\title{
A MAXIMUM PRINCIPLE FOR HYPERBOLIC EQUATIONS IN A NEIGHBORHOOD OF AN INITIAL LINE $\left({ }^{1}\right)$
}

\author{
BY \\ M. H. PROTTER
}

1. Introduction. Germain and Bader [3] were the first to find that a maximum principle could be stated for hyperbolic equations. They showed that such a maximum principle was valid for the Tricomi equation

$$
y u_{x x}+u_{y y}=0, \quad y<0
$$

among the class of solutions which vanish on one characteristic.

This property was extended by Agmon, Nirenberg and Protter [1] to equations of the form

$$
K(x, y) u_{x x}+u_{y y}+\alpha u_{x}+\beta u_{y}+u=0, \quad K<0
$$

provided the coefficients satisfied certain basic inequalities. The maximum principle concerned solutions of (2) bounded by two characteristic arcs, say $A B$ and $A C$, and a noncharacteristic "space-like" curve joining $B$ and $C$. If $u(x, y)$ is a solution of (1) which vanishes along the characteristic $A B$ and if the coefficients of (1) satisfy certain inequalities (valid for (1)) then the maximum of $u$ in $A B C$ occurs on the noncharacteristic $B C$.

Recently Weinberger [4] obtained a maximum principle for a class of hyperbolic equations in which hypotheses on the solutions are imposed along non-characteristic curves rather than characteristic curves. We write the equation in the form

$$
L u \equiv\left(a u_{x}\right)_{x}-\left(b u_{y}\right)_{y}+c u_{x}+d u_{y}+f u=0, \quad a>0, b>0
$$

and consider a curvilinear triangle $T$ bounded by a segment $A B$ of the $x$-axis and the characteristics $A C$ and $B C$ extending into $y>0$. Weinberger showed that if the coefficients of (3) satisfied certain inequalities and if we consider the class of solutions $\{u\}$ such that $\partial u / \partial y \leqq 0$ on $A B$, then the maximum of $u$ in $T$ must occur on $A B$. The principal restriction on the coefficients in (3) required by Weinberger is the condition:

$$
\left.\begin{array}{l}
b^{1 / 2}\left(\frac{\partial}{\partial y}\left((a b)^{1 / 2}\right)+c\right)+a^{1 / 2}\left(-\frac{\partial}{\partial x}\left((a b)^{1 / 2}\right)+d\right) \geqq 0 \\
b^{1 / 2}\left(\frac{\partial}{\partial y}\left((a b)^{1 / 2}\right)-c\right)+a^{1 / 2}\left(\frac{\partial}{\partial x}\left((a b)^{1 / 2}\right)+d\right) \geqq 0
\end{array}\right\} \text { in } T .
$$

Received by the editors July 22, 1956.

(1) This research was supported by the United States Air Force through the Air Force Office of Scientific Research of the Air Research and Development Command under Contract No. AF 18(600)-1117. 
These conditions are shown to be "best possible" in the sense that examples are given where the maximum principle does not hold as soon as (4) is violated.

It is the purpose of this paper to establish a maximum principle for hyperbolic equations which is independent of condition (4). In view of the examples of the "best possible" nature of condition (4), it is clear that the maximum principle will take a somewhat different form. It will be shown that there is a constant $y_{0}$ such that for certain classes of solutions of (3) the maximum of $u$ in the part of $T$ such that $0 \leqq y \leqq y_{0}$ occurs for $y=0$. For the case where (4) holds the line $y=y_{0}$ passes through $C$ and the maximum principle is valid in $T$, yielding Weinberger's result.

Bochner [2] has shown that the ultraspherical polynomials satisfy a certain maximum principle. Suppose $\sum_{n=0}^{\infty} a_{n} P_{n}(x) \leqq 0,-1 \leqq x \leqq 1$; then it follows that $\sum_{n=0}^{\infty} a_{n} P_{n}(x) P_{n}(y) \leqq 0,-1 \leqq x \leqq 1,-1 \leqq y \leqq 1$. Since $P_{n}(x) P_{n}(y)$ satisfies a certain (singular) hyperbolic equation Weinberger showed that his maximum principle can be adjusted to the singular case to yield Bochner's result. In $\$ 3$ it is shown how the singular behavior affects the maximum theorem in the general case. In addition certain other applications to ordinary differential equations are established.

2. The main theorems. We consider not only the operator $L u$ defined by (3) but also the adjoint operator

$$
M v \equiv\left(a v_{x}\right)_{x}-\left(b v_{y}\right)_{y}-(c v)_{x}-(d v)_{y}+f v .
$$

The coefficients are functions of class $C^{\prime}$ in the domain under consideration and the characteristics $A C$ and $B C$ bounding $T$ are solutions of the ordinary equations

$$
d y / d x= \pm(b / a)^{1 / 2}
$$

We apply Green's theorem to obtain the identity

$$
\begin{aligned}
\iint_{T}(v L u-u M v) d x d y \\
=\oint_{S}\left\{a v u_{x} d y+b v u_{y} d x+c u v d y-d u v d x-a u v_{x} d y-b u v_{y} d x\right\}
\end{aligned}
$$

where $S$ is the boundary of $T$. Making use of the equations for the characteristics (6), we obtain

$$
\begin{aligned}
\iint_{T}(v L u-u M v) d x d y= & \int_{A}^{B}\left[b\left(v u_{y}-u v_{y}\right)-d u v\right] d x \\
& +\int_{B}^{C}\left[-(a b)^{1 / 2} \imath d u+(a b)^{1 / 2} u d v+u v(c d y-d d x)\right] \\
& +\int_{C}^{A}\left[(a b)^{1 / 2} v^{\prime} d u-(a b)^{1 / 2} u d v+u v(c d y-d d x)\right] .
\end{aligned}
$$


An integration by parts in the last two equations on the right, and a rearrangement yields

$$
\begin{aligned}
& 2(a(C) b(C))^{1 / 2} u(C) v(C) \\
& =(a(A) b(A))^{1 / 2} u(A) v(A)+(a(B) b(B))^{1 / 2} u(B) v(B)+\iint_{T}(u M v-v L u) d x d y \\
& \quad+\int_{A}^{B}\left[b\left(v u_{y}-u v_{y}\right)-d u v\right] d x \\
& (7)+\int_{B}^{C} u\left[2(a b)^{1 / 2} d v+v\left\{\left((a b)^{1 / 2}\right)_{x} d x+\left((a b)^{1 / 2}\right)_{y} d y+c d y-d d x\right\}\right] \\
& \quad+\int_{A}^{C} u\left[2(a b)^{1 / 2} d v+v\left\{\left((a b)^{1 / 2}\right)_{x} d x+\left((a b)^{1 / 2}\right)_{y} d y-c d y+d d x\right\}\right] .
\end{aligned}
$$

We define the function

$$
v(x, y)=e^{\gamma x}\left(1-\beta e^{-\alpha y}\right), \quad \gamma \geqq 0, \alpha \geqq 0,0 \leqq \beta<1
$$

and compute the expression $M v$ :

$$
\begin{aligned}
M v= & e^{\gamma x-\alpha y}\left(e^{\alpha y}-\beta\right)\left[a \gamma^{2}+\gamma a_{x}-c \gamma+\left(f-c_{x}-d_{y}\right)\right] \\
& +e^{\gamma x-\alpha y}\left[\alpha^{2} \beta b-b_{y} \alpha \beta-d \alpha \beta\right] .
\end{aligned}
$$

We select $\alpha$ and $\gamma$ so large that $M v \geqq 0$. We note that for sufficiently large $\alpha$ we can make $M v \geqq 0$ for any fixed $\gamma$ for $0 \leqq y \leqq y_{0}$ if $y_{0}$ is sufficiently small and $\beta$ is sufficiently close to unity.

We now consider the inequalities

$$
\begin{gathered}
-2 a v_{x}+2(a b)^{1 / 2} v_{y}+v\left[-(a / b)^{1 / 2}\left((a b)^{1 / 2}\right)_{x}+\left((a b)^{1 / 2}\right)_{y}+c+d(a / b)^{1 / 2}\right] \geqq 0, \\
2 a v_{x}+2(a b)^{1 / 2} v_{y}+v\left[(a / b)^{1 / 2}\left((a b)^{1 / 2}\right)_{x}+\left((a b)^{1 / 2}\right)_{y}-c .+d(a / b)^{1 / 2}\right] \geqq 0 .
\end{gathered}
$$

If necessary we further increase $\alpha$, decrease $y_{0}$ and make $\beta$ closer to unity so that both inequalities in (9) as well as $M v \geqq 0$ hold in the common part of $T$ and $0 \leqq y \leqq y_{0}$. We denote this region by $D$.

Theorem 1. Let Lu $\geqq 0$ in $D$ and suppose $f \geqq 0$ in $D, d(x, 0) \leqq 0$. If $u(x, 0)$ $\leqq 0$ on $A B$ and $(u / v)_{v} \leqq 0$ on $A B$ with $v(x, y)$ defined by $(8)$, then the maximum of $u$ in $D$ occurs on $A B$.

Proof. We first consider the case $u(x, 0)<0,(u / v)_{y} \leqq 0$. Suppose the maximum of $u$ occurs at a point $Q$ in the interior of $D$. If $u(Q) \geqq 0$ then there is a point $Q^{\prime}$ closest to $A B$ where $u\left(Q^{\prime}\right)=0$. If the maximum of $u$ (occurring at $Q$ ) is less than zero we add a constant $N>0$ to $u$ so that the function $u_{1}=u+N$ has zero as its maximum at $Q$. A simple computation shows that $L u_{1} \geqq 0$ and $\left(u_{1} / v\right)_{y} \leqq 0$ on $A B$, and of course $u_{1} \leqq 0$ on $A B$ if its maximum $(=0)$ occurs at $Q$. Construct the characteristics $\gamma_{1}$ and $\gamma_{2}$ of (6) through $Q$ or $Q^{\prime}$, which- 
ever is applicable, and denote the intersections with $A B$ by $P_{1}$ and $P_{2}$. Applying (7) to the curvilinear triangle $D^{\prime}$ bounded by $P_{1} P_{2} Q^{\prime}$ (or $P_{1} P_{2} Q$ ) we find

$$
\begin{aligned}
0= & \left(a\left(P_{1}\right) b\left(P_{1}\right)\right)^{1 / 2} v\left(P_{1}\right) u\left(P_{1}\right)+\left(a\left(P_{2}\right) b\left(P_{2}\right)\right)^{1 / 2} v\left(P_{2}\right) u\left(P_{2}\right) \\
& +\iint_{D^{\prime}}(u M v-v L u) d x d y+\int_{P_{1}}^{P_{2}}\left[b\left(v u_{y}-u v_{y}\right)-d u v\right] d x \\
& +\int_{P_{2}}^{Q} u\left[d\left((a b)^{1 / 2} v\right)+v(c d y-d d x)+(a b)^{1 / 2} d v\right] \\
& +\int_{P_{1}}^{Q} u\left[d\left((a b)^{1 / 2} v\right)-v(c d y-d d x)+(a b)^{1 / 2} d v\right] .
\end{aligned}
$$

All the terms on the right side of the above expression are nonpositive and hence each must vanish. This can only happen if $u \equiv 0$. Thus the maximum of $u$ occurs on $A B$.

For the general case we define $w=1-e^{\delta y}$ and consider the function $u_{1}$ $=u+w$. Then $L w>0$ in $D$ for sufficiently large $\delta, u_{1}(x, 0)=u(x, 0)$ and $\left(u_{1} / v\right)_{y}=(u / v)_{y}-\delta / v<0$ for $y=0$. Thus the function $u_{2}=u_{1}-\epsilon$ with $\epsilon$ a sufficiently small positive constant will satisfy the conditions under which the result has already been established. Hence the maximum of $u_{2}$ occurs on $A B$. Letting $\epsilon \rightarrow 0$ we see that the same must be true for $u_{1}$. Since $u(x, y) \leqq u_{1}(x, y)$ in $D$ and $u(x, 0)=u_{1}(x, 0)$ the maximum of $u$ occurs on $A B$.

In the applications it is often important to include the cases where the equation becomes singular on the initial line. If, for example $a(x, 0)=0$ for $y=0$ the results of Theorem 1 remain valid. However if $b=0$ for $y=0$ it may or may not happen that a choice of $\alpha, \beta$ and $\gamma$ can be made so that inequalities (9) as well as $M v \geqq 0$ hold. If these constants can be so chosen then we have the following theorem.

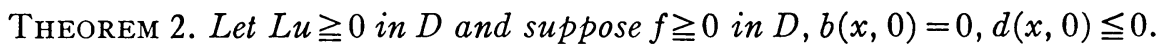
If $\alpha, \beta$ and $\gamma$ can be so chosen that $M v \geqq 0$ and inequalities (9) hold in $D$ and if $u(x, 0) \leqq 0$ on $A B$, then the maximum of $u$ in $D$ occurs on $A B$.

Proof. The argument here is similar to that given in the proof of Theorem 1. We note that the second integral in (10) reduces to

$$
-\int_{P_{1}}^{P_{2}} d u v d x
$$

and thus the condition $(u / v)_{y} \leqq 0$ which appears in Theorem 1 is eliminated.

In the special case $c=d=0$ and $b \sim y^{\xi}, 0<\xi<2$ as $y \rightarrow 0$ it can be shown that a selection of $\alpha, \beta$ and $\gamma$ can always be made. We consider the operator

$$
L_{1} u \equiv\left(a u_{x}\right)_{x}-\left(b u_{y}\right)_{y}+f u
$$

and show that the inequalities $L_{1} v \geqq 0$ and (9) hold. It is clear that if $\alpha=\beta=0$ 
and $\gamma$ is sufficiently large then $L_{1} v \geqq 0$. Also in (9) the quantity $(a)^{1 / 2}\left((b)^{1 / 2}\right)_{y}$ is dominant and hence (9) will hold in some neighborhood $D_{1}$ of the initial line. Thus we have

TheOREM 3. If $L_{1} u \geqq 0$ and $f \geqq 0$ in $D_{1}$ and if $u(x, 0) \leqq 0$ on $A B$ then the maximum of $u$ in $D$ occurs on $A B$.

Theorem 3 is the one which will be applied to singular ordinary differential equations, the singularity corresponding to the condition $b=0$.

If $f \equiv 0$ in Theorem 3 then the maximum theorem holds without the condition $u \leqq 0$ as a sufficiently large positive constant may be subtracted.

CoRlllary 1. Let $L_{1} u \geqq 0$ in $D_{1}$ and suppose $f \equiv 0$ in $D$. Then the maximum of $u$ in $D_{1}$ occurs on $A B$.

If $\beta=\gamma=0$ the function $v(y) \equiv 1$ suffices; and if the additional conditions (4) of Weinberger are assumed the theorems become those given in [4].

The result of Theorem 1 implies that the Riemann function of $L u$ is non-negative in $D$.

3. Ordinary differential equations. We consider the ordinary differential equations

$$
\begin{array}{llrl}
\left(f_{1}(x) \phi^{\prime}(x)\right)^{\prime}+g_{1}(x) \phi(x)=0, & f_{1}(x)>0, & 0 \leqq x \leqq x_{0}, \\
\left(f_{2}(y) \psi^{\prime}(y)\right)^{\prime}+g_{2}(y) \psi(y)=0, & f_{2}(y)>0, & 0 \leqq y \leqq x_{0} / 2 .
\end{array}
$$

The function $u(x, y)=\phi(x) \psi(y)$ satisfies the hyperbolic equation

$$
\begin{aligned}
L u \equiv & \left(f_{1}(x) f_{2}(y) u_{x}\right)_{x}-\left(f_{1}(x) f_{2}(y) u_{y}\right)_{y} \\
& +\left(g_{1}(x) f_{2}(y)-f_{1}(x) g_{2}(y)\right) u=0 .
\end{aligned}
$$

We shall suppose that

$$
g_{1}(x) f_{2}(y)-f_{1}(x) g_{2}(y) \geqq 0, \quad 0 \leqq x \leqq x_{0}, \quad 0 \leqq y \leqq x_{0} / 2
$$

so that the maximum principle of Theorem 1 applies to equation (13). If we select $\gamma=0$ in the determination of $v$ conditions (9) become

$$
\alpha \beta e^{-\alpha y}+\left(1-\beta e^{-\alpha y}\right)\left[\frac{f_{2}^{\prime}(y)}{f_{2}(y)}-\frac{\left|f_{1}^{\prime}(x)\right|}{f_{1}(x)}\right] \geqq 0
$$

while the condition $M v \geqq 0$ is, in virtue of (14),

$$
\alpha^{2} \geqq \frac{\alpha f_{2}^{\prime}(y)}{f_{2}(y)} .
$$

Theorem 1 now yields a comparison theorem on the distance between zeros of ordinary differential equations.

Theorem 4. Suppose $f_{i}, g_{i}, i=1,2$ satisfy (13) and $\beta$ and $y_{0}$ are chosen so that (15) and (16) hold for $0 \leqq x \leqq x_{0}, 0 \leqq y \leqq y_{0} \leqq x_{0} / 2$. If $\phi(x)$ is a positive 
solution of (11) in $0 \leqq x \leqq x_{0}$, then the solution $\psi(y)$ of (12) satisfying the intial conditions $\psi(0)=0, \psi^{\prime}(0)<0$ has no zeros in the interval $0<y<y_{0}$.

Proof. The function $u=\phi(x) \psi(y)$ satisfies (13) with initial conditions $u(x, 0) \equiv 0, u_{y}(x, 0)=\psi^{\prime}(0) \phi(x)<0,0 \leqq x \leqq x_{0}$. The condition $(u / v)_{y} \leqq 0$ is $v u_{y}-u v_{y}=v u_{y} \leqq 0$ and is automatically satisfied for any non-negative function $v$. Therefore Theorem 1 applies, the maximum of $u$ occurs for $y=0$, and hence $u<0$ in the domain $D$ defined in Theorem 1 .

An interesting special case occurs if $f_{1}(x) \equiv 1$ and $g_{1}(x)=\lambda^{2}$. The distance between zeros for (11) is thus $\pi / \lambda$ and (14), (15) become

$$
\lambda^{2} f_{2}(y) \geqq g_{2}(y), \quad \alpha \beta e^{-\alpha y}+\left(1-\beta e^{-\alpha y}\right) \frac{f_{2}^{\prime}(y)}{f_{2}(y)} \geqq 0
$$

while (16) remains unchanged.

CoRollary 2. If $\lambda^{2} f_{2}(y) \geqq g_{2}(y)$ and $\alpha>0,0<\beta<1$ are such that $\alpha \beta e^{-\alpha y}$ $+\left(1-\beta e^{-\alpha y}\right) f_{2}^{\prime}(y) / f_{2}(y) \geqq 0$ for $0 \leqq y \leqq y_{0} \leqq \pi / 2 \lambda$, the solution $\psi(y)$ of $(12)$ with initial conditions $\psi(0)=0, \psi^{\prime}(0)=\xi<0$ has no zero in the interval $0<y<y_{0}$.

In the special case $\lambda=1, f_{2}=g_{2}, f_{2}^{\prime}(y) \geqq 0$ we can select $\alpha=0$. Then Corollary 3 states that solutions of the equation

$$
\left(f_{2}(y) \psi^{\prime}\right)^{\prime}+f_{2} \psi=0
$$

with $f_{2}>0, f_{2}^{\prime} \geqq 0$ have minimum distance $\pi / 2$ between zeros. This result was obtained by Weinberger [4].

Because of the special nature of the functions involved it is possible to obtain other estimates for the distance between zeros of solutions of (12) in the case $f_{1}(x) \equiv 1, g_{1}(x)=\lambda^{2}$. For example, select for $v$ the function

$$
v(y)=1 / f_{2}(y) \text {. }
$$

Then condition (9) is automatically satisfied, while (14) and (16) become, respectively,

$$
\lambda^{2} f_{2}(y) \geqq g_{2}(y), \quad\left(f_{2}^{\prime} / f_{2}\right)^{\prime} \geqq 0 .
$$

CorollaRy 3. If $\lambda^{2} f_{2}(y) \geqq g_{2}(y), f_{2}(y) f_{2}^{\prime \prime}(y) \geqq\left[f_{2}^{\prime}(y)\right]^{2}$ for $0 \leqq y \leqq y_{0} \leqq \pi / 2 \lambda$, the solution $\psi(y)$ of $(12)$ with initial conditions $\psi(0)=0, \psi^{\prime}(0)=\xi<0$ has no zero in the interval $0 \leqq y \leqq y_{0}$.

Again considering the case $f_{1}(x) \equiv 1, g_{1}(x)=\lambda^{2}$ in (11) we set

$$
v=\int_{0}^{y} \frac{1}{f_{2}(y)} d y \text {. }
$$

Then we have condition (14)

$$
\lambda^{2} f_{2}(y) \geqq g_{2}(y)
$$


and $M v \geqq 0$ in virtue of the above condition and the definition of $v$. Conditions (9) become

$$
1+f_{2}^{\prime}(y) \int_{0}^{y} \frac{1}{f_{2}(y)} d y \geqq 0
$$

Corollary 4. If

$$
\lambda^{2} f_{2}(y) \geqq g_{2}(y), \quad 1 \geqq-f_{2}^{\prime}(y) \int_{0}^{y} \frac{1}{f_{2}(y)} d y \quad 0 \leqq y \leqq y_{0} \leqq \pi / 2 \lambda,
$$

the solution $\psi(y)$ of (12) with initial conditions $\psi(0)=0, \psi^{\prime}(0)=\xi<0$ has no zero in the interval $0 \leqq y \leqq y_{0}$.

In Corollaries 2, 3 and 4 we see that $\lambda$ can always be selected large enough so that $\lambda^{2} f_{2} \geqq g_{2}$. Corollaries 2 and 4 have the property that they yield a minimum distance between zeros regardless of the behavior of the derivative of $f_{2}(y)$. Corollary 3, however, is of a more special character and yields a result only if $f_{2}(y) f_{2}^{\prime \prime}(y) \geqq\left(f_{2}^{\prime}(y)\right)^{2}$ in some interval.

Further theorems on ordinary differential equations are obtained from the observation that the function $u=\phi(x) \psi(y)$, with $\phi, \psi$ solutions of (11) and (12) respectively, satisfies the equation

$$
L u \equiv\left(f_{1}(x) g_{2}(y) u_{x}\right)_{x}-\left(f_{2}(y) g_{1}(x) u_{y}\right)_{y}=0
$$

which is hyperbolic if the functions $f_{i}, g_{i}, i=1,2$, are positive. In this form the condition (14) disappears (and is replaced by the conditions that $g_{1}, g_{2}$ be positive). For $v=1-\beta e^{-\alpha y}$ we find

$$
L v=\alpha \beta e^{-\alpha y} g_{1}\left(\alpha f_{2}-f_{2}^{\prime}\right)
$$

and conditions (9) become

$$
\alpha \beta e^{-\alpha y}+\left(1-\beta e^{-\alpha y}\right)\left[-\left|\frac{1}{2 g_{1}}\left(\frac{g_{2}}{f_{1} f_{2} g_{1}}\right)^{1 / 2}\left(f_{1} g_{1}\right)_{x}\right|+\frac{\left(f_{2} g_{2}\right)_{y}}{2\left(f_{2} g_{2}\right)}\right] \geqq 0 .
$$

The characteristics of (19) are solutions of

$$
\frac{d y}{d x}= \pm\left(\frac{g_{1}(x) f_{2}(y)}{f_{1}(x) g_{2}(y)}\right)^{1 / 2}
$$

The limitations on the domain in which the maximum principle holds involve not only (21) but also the size on the characteristic triangle as determined by (22).

Theorem 5. Suppose $f_{i}, g_{i}, i=1,2$ are positive and $\alpha, \beta$ and $y_{0}$ are chosen so that (20) and (21) hold for $0 \leqq x \leqq x_{0}, 0 \leqq y \leqq y_{0}$; let $y_{0} \leqq \bar{y}_{0}\left(x_{0}\right)$ where $\bar{y}_{0}\left(x_{0}\right)$ is the height of the characteristic triangle. If $\phi(x)$ is a positive solution of (11) in $0 \leqq x \leqq x_{0}$, then the solution $\psi(y)$ of (12) satisfying the initial conditions $\psi(0)=0$, 
$\psi^{\prime}(0)<0$ has no zero in the interval $0<y<y_{0}$.

As in the case of Theorem 4 , if we select $f_{1}(x) \equiv 1, g_{1}(x) \equiv \lambda^{2}$ we obtain corollaries analogous to Corollaries 2, 3, and 4 .

Corollary 5. If $\alpha, \beta, y_{0}$ are chosen so that

$$
\alpha \beta e^{-\alpha y}+\frac{1}{2}\left(1-\beta e^{-\alpha y}\right) \frac{\left(f_{2} g_{2}\right)^{\prime}}{f_{2} g_{2}} \geqq 0
$$

for $0 \leqq y \leqq y_{0}$, with $y_{0} \leqq \bar{y}\left(x_{0}\right)$, then the solution $\psi(y)$ of (12) satisfying initial conditions $\psi(0)=0, \psi^{\prime}(0)<0$ has no zero in the interval $0<y<y_{0}$. The quantity $\bar{y}_{0}\left(x_{0}\right)$ is the height of the characteristic triangle determined by the curves

$$
x= \pm \int\left(\lambda^{2} f_{2}(y) / g_{2}(y)\right)^{1 / 2} d y+c .
$$

CoROllary 6. If $f_{2}(y) f_{2}^{\prime \prime}(y) \geqq\left(f_{2}^{\prime}(y)\right)^{2}, 0 \leqq y \leqq y_{0}, y_{0} \leqq \bar{y}\left(x_{0}\right)$, then the result of Corollary 5 holds.

Corollary 7. If $1 \geqq-f_{2}^{\prime}(y) \int_{0}^{y}\left(1 / f_{2}(y)\right) d y, 0 \leqq y \leqq y_{0}, y_{0} \leqq \bar{y}\left(x_{0}\right)$, then the result of Corollary 5 holds.

We now consider the equation

$$
\left(p(y) \psi^{\prime}\right)^{\prime}+q(y) \psi=0, \quad \quad p \geqq 0
$$

in the interval $0 \leqq y \leqq y_{0}$, where $p(0)=0$ and $p(x) / q(x)$ has a zero of order not exceeding 2 at $x=0$. Many of the equations of mathematical physics have this form and if $p, q$ are analytic, there is one solution analytic in the neighborhood of the origin and satisfying the condition $\psi(0)=1$. This condition on analyticity replaces the condition on the derivative $\psi^{\prime}(0)$. Under these circumstances Theorem 3 yields results on the distance between zeros.

We consider the equations

$$
\begin{aligned}
\frac{d}{d x}\left(p(x) \frac{d \phi}{d x}\right)+q(x) \phi & =0 \\
\frac{d}{d y}\left(p(y) \frac{d \psi}{d y}\right)+q(y) \psi(y) & =0
\end{aligned}
$$

where $p(y)>0,0<y \leqq y_{0}, p(0)=0$; the function $u(x, y)=\phi(x) \psi(y)$ satisfies the hyperbolic equation

$$
\left(p(x) q(y) u_{x}\right)_{x}-\left(p(y) q(x) u_{y}\right)_{y}=0 .
$$

The characteristics are solutions of

$$
\left(\frac{q(y)}{p(y)}\right)^{1 / 2} d y= \pm\left(\frac{q(x)}{p(x)}\right)^{1 / 2} d x
$$


so that one of the characteristic curves is the line $y=x$ while another is $\int_{0}^{y}(q(y) / p(y))^{1 / 2} d y=\int_{x_{0}}^{x}(q(x) / p(x))^{1 / 2} d x$.

For $v=1-\beta e^{-\alpha y}$ conditions (9) become

$$
\begin{aligned}
\alpha \beta e^{-\alpha y}+\left(1-\beta e^{-\alpha y}\right)\left[-\mid \frac{1}{2 q(x)}\left(\frac{q(y)}{p(x) p(y) q(x)}\right)^{1 / 2}(\right. & p(x) q(x))_{x} \mid \\
& \left.+\frac{(p(y) q(y))_{y}}{2 p(y) q(y)}\right] \geqq 0 .
\end{aligned}
$$

Theorem 3 yields the following result.

THEOREM 6. Suppose $p, q$ are analytic and $p / q$ has a zero of order not greater than 2 at the origin. Let $\alpha, \beta$, and $y_{0}$ be chosen so that (26) holds for $0 \leqq x \leqq x_{0}$, $0 \leqq y \leqq y_{0}$ with $y_{0} \leqq \bar{y}\left(x_{0}\right)$. If $\phi(x)$ is a positive solution of (23) in $0 \leqq x \leqq x_{0}$, then the solution $\psi(y)$ of (24) satisfying the initial condition $\psi(0)=\xi>0$ has no zero in the interval $0 \leqq y \leqq y_{0}$.

Proof. The result follows immediately from Theorem 3 since equation (25) has coefficients $a=p(x) q(y), b=p(y) q(x), c=d=f=0$. We note that $b(x, 0)=0$ and $u(x, 0)=\phi(x) \psi(0)$ is positive. Hence the minimum of $u(x, y)$ occurs on the $x$-axis and $\psi(y)$ can have no zero for $0 \leqq y \leqq y_{0}$.

Theorem 6 may be used to extend a result of Weinberger [4] concerning solutions of singular differential equations which itself is an extension of a result of Bochner's obtained for sums of ultraspherical polynomials.

For the equation

$$
\frac{d}{d y}\left(p(y) \frac{d \psi}{d y}\right)+\{\lambda q(y)-r(y)\} \psi(y)=0
$$

we can pose an eigenvalue problem by adding an additional boundary condition. If $\left\{\lambda_{n}\right\}$ is a sequence of eigenvalues, $\psi_{n}(y)$ the corresponding eigenfunctions, and similarly for

$$
\frac{d}{d x}\left(p(x) \frac{d \phi}{d x}\right)+\{\lambda q(x)-r(x)\} \phi(x)=0
$$

then the quantity $u=\sum_{n=0}^{\infty} a_{n} \phi_{n}(x) \psi_{n}(y)$ satisfies the equation

$$
\left(p(x) q(y) u_{x}\right)_{x}-\left(q(x) p(y) u_{y}\right)_{y}+(q(x) r(y)-q(y) r(x)) u=0 .
$$

If $p$ and $q$ are each of one sign in the domain under consideration, Equation (29) satisfies a maximum principle in a certain subdomain whenever $q(x) r(y)$ $-q(y) r(x) \geqq 0$. We note the important fact that the quantity $\lambda$ does not appear in (29). Thus while $\phi_{n}(x), \psi_{n}(y), n=1,2, \cdots$, satisfy equations (27), (28) respectively which change with $n$, the product $\phi_{n}(x) \psi_{n}(y)$ satisfies (29) for all $n$. If we select initial values of $(27)$ to be $\psi_{n}(0)=1$, then any linear 
combination $\sum a_{n} \phi_{n}(x) \psi_{n}(y)$ satisfies (29) with initial value $\sum a_{n} \phi_{n}(x)$.

Let $C_{n}(x)$ be the eigenfunctions of

$$
\frac{d}{d x}\left[\left(1-x^{2}\right)^{(\nu+1 / 2)} \frac{d \phi}{d x}\right]+n(n+2 \nu)\left(1-x^{2}\right)^{\nu-1 / 2} \phi=0, \quad \nu \geqq 0
$$

normalized so that $C_{n}^{\nu}(1)=1$. Bochner [2] showed that if $\sum_{n=0}^{\infty} a_{n} C_{n}^{\nu}(x) \geqq 0$, $-1 \leqq x \leqq 1$ then

$$
\sum_{n=0}^{\infty} a_{n} C_{n}^{\nu}(x) C_{n}^{\nu}(y) \geqq 0, \quad-1 \leqq x \leqq 1,-1 \leqq y \leqq 1 .
$$

Equation (30) is of the form (27) with $r=0$, and the initial line situated along the line $y=1$ instead of $y=0$ and a singularity at $x=1$ instead of $x=0$. The maximum principle of Theorem 5 may now be applied to (29) with $p=\left(1-x^{2}\right)^{\nu+1 / 2}, q=\left(1-x^{2}\right)^{\nu-1 / 2}, r=0$. The initial values along the line $y=1$ are $\sum_{n=0}^{\infty} a_{n} \phi_{n}(x),-1 \leqq x \leqq 1$. If this is positive, then the maximum principle holds in the characteristic triangle below $y=1$ if condition (21) holds. This is

$$
\alpha \beta e^{+\alpha(y-1)}+\frac{2\left(1-\beta e^{+\alpha(y-1)}\right)}{\left(1-y^{2}\right)^{1 / 2}}\left[\frac{-\nu y}{\left(1-y^{2}\right)^{1 / 2}}+\left|\frac{\nu x}{\left(1-x^{2}\right)^{1 / 2}}\right|\right] \leqq 0 .
$$

This relation holds for $\alpha=0$ if $\nu \geqq 0$ and

$$
|y| \geqq|x| \text {, }
$$

that is, in the characteristic triangle. By symmetry this extends to the entire square. Note that this result cannot be extended to negative values of $\nu$ regardless of the choice for $\alpha$ because of the singularity at $y=1$.

A maximum principle can be stated for other functions that occur in the equations of mathematical physics. For example, if $J_{n}\left(\lambda_{k n} x\right)$ are the Bessel functions of order $n$ with $\left\{\lambda_{k n}\right\}$ determined as usual by the zeros, we recall that the equation

$$
x \frac{d^{2}}{d x^{2}} J_{n}\left(\lambda_{k n} x\right)+\frac{d}{d x} J_{n}\left(\lambda_{k n} x\right)+\left(\lambda_{k n}^{2} x-\frac{n^{2}}{x}\right) J_{n}\left(\lambda_{k n} x\right)=0
$$

holds. We consider such equations for fixed $n$ and varying values of $k$. We again obtain an equation of the form (29) with $p=x, q=x, r=n^{2} / x$. Then a maximum principle holds if $n^{2}(x / y)-n^{2}(y / x) \geqq 0$ or $x^{2}-y^{2} \geqq 0$ if $x, y$ are nonnegative and if conditions (9) hold. They are

$$
x y \alpha \beta e^{-\alpha y}+\left(1-\beta e^{-\alpha y}\right)[x-|y|] \geqq 0 .
$$

Thus a maximum principle holds for expressions of the form

$$
\sum_{k} a_{k} J_{n}\left(\lambda_{k n} x\right) J_{n}\left(\lambda_{k n} y\right)
$$

in appropriate domains. 


\section{BibliogRAPHY}

1. S. Agmon, L. Nirenberg, and M. H. Protter, A maximum principle for a class of hyperbolic equations and applications to equations of mixed elliptic-hyperbolic type, Communications on Pure and Applied Mathematics vol. 6 (1953) pp. 455-470.

2. S. Bochner, Positive zonal functions on spheres, Proc. Nat. Acad. Sci. vol. 40 (1954) pp. 1141-1147.

3. P. Germain and R. Bader, Sur quelques problémes à l'equation de type mixte de Tricomi, Office Nationale d'Etudes Recherches Aéronautiques, Publication No. 54, 1952.

4. H. F. Weinberger, A maximum property of Cauchy's problem, Ann. of Math. vol. 64 (1956) pp. 505-513.

\section{University of California}

Berkeley, Cai.jfornia 